\title{
Habilidades metacognitivas y su relación con variables de género y tipo de desempeño profesional de una muestra de docentes colombianos
}

Metacognitive Skills and Their Relationship with Gender, Educational Level, and Professional PerformanceVariables of a Sample of Colombian Teachers

Habilidades metacognitivas e sua relação comvariáveis de gênero e tipo de desempenho profissional de uma amostra de professores colombianos

Antonio Gutiérrez de Blume* (iD) orcid.org/0000-0001-6809-1728

Diana Marcela Montoya-Londoño** (iD) orcid.org/0000-0001-8007-0102

Andrea Milena 0sorio-Cárdenas*** (D) orcid.org/0000-0002-5143-2829

\section{Para citar este artículo}

Gutiérrez de Blume, A., Montoya-Londoño, D. y Osorio-Cárdenas, A. (2022). Habilidades metacognitivas y su relación con variables de género y tipo de desempeño profesional de una muestra de docentes colombianos. Revista Colombiana de Educación, 1(84), 1-23. https://doi.org/10.17227/rce.num84-11298.

\section{(ㅇ) $(1)(9$}




\section{Resumen}

\author{
Palabras clave: \\ metacognición; nivel de \\ conocimiento; conciencia; \\ base de conocimientos de \\ enseñanza; aprendizaje; \\ evaluación del docente
}

El objetivo de este artículo de investigación fue establecer las diferencias en el conocimiento metacognitivo (declarativo, de procedimiento y condicional) y la regulación (planificación, monitoreo y evaluación) en una muestra de docentes colombianos ( $N=771)$ en función de las variables de género y tipo de desempeño profesional. Se realizó un análisis de varianza factorial multivariante (Manova) para examinar el efecto de género (hombre, mujer) y tipo de desempeño profesional (estudiante universitario de pregrado en educación de último nivel de práctica, en los ciclos de básica y media académica; docente titular en los niveles de básica y media académica; y docente universitario), en las diversas habilidades metacognitivas (conocimiento declarativo, conocimiento de procedimiento, conocimiento condicional, planificación, monitoreo y evaluación). Los resultados indicaron que hubo diferencias significativas entre los docentes en función del nivel de formación y el género. Las mujeres reportaron habilidades de regulación significativamente más altas (monitoreo y evaluación), en comparación con los hombres. Con respecto al nivel de formación, las diferencias significativas fueron evidentes para el conocimiento condicional, la planificación y el monitoreo. Se discuten las implicaciones para la teoría, la investigación y la práctica considerando los hallazgos encontrados.

\section{Abstract}

The purpose of the present research article was to examine differences in metacognitive knowledge (declarative, procedural, and conditional) and regulation (planning, monitoring, and evaluation) in a sample of Colombian teachers ( $N=$ 771) based on gender and type of professional practice. A factorial multivariate analysis of variance (MANOvA) was conducted to examine the effect of gender (male, female) and type of professional practice (university student studying elementary and middle education, in-service teacher in the levels of elementary and middle-level education, and university professor) on various metacognitive skills (declarative knowledge, procedural knowledge, conditional knowledge, planning, monitoring, and evaluation). Results indicated that there were significant differences between teachers according to professional practice and gender. Females reported significantly higher regulatory skills (monitoring and evaluation) compared to males. Regarding professional practice, the significant differences were evident for conditional knowledge, planning, and monitoring. Implications for theory, research, and practice are discussed considering our findings.

\section{Resumo}

0 objetivo do presente artigo de pesquisa foi estabelecer as diferenças no conhecimento metacognitivo (declarativo, procedimental e condicional) e a regulação (planejamento, monitoramento e avaliação) em uma amostra de professores colombianos ( $N=771$ ) em função das variáveis de gênero e tipo de desempenho profissional. Realizou-se uma análise de variância fatorial multivariada (MANOVA) para examinar o efeito do género (masculino, feminino) e tipo de desempenho profissional (estudante universitário de graduação em educação no último nível de estágio, nos ciclos de ensino fundamental e médio; professor efetivo nos níveis fundame ntal e médio; e docente universitário), nas diversas habilidades metacognitivas (conhecimento declarativo, conhecimento de procedimento, conhecimento condicional, planejamento, monitoramento e avaliação). Os resultados indicam que houve diferenças significativas entre os docentes em função do nível educacional de formação e gênero. As mulheres relataram habilidades de regulação significativamente mais altas (monitoração e avaliação), em comparação com os homens. Com relação ao nível educacional de formação, as diferenças significativas foram evidentes para o conhecimento condicional, o planejamento e o monitoramento. Discutese as implicações para a teoria, a investigação e a prática considerando as descobertas encontradas. 


\section{Introducción}

La metacognición se asume como un constructo que da cuenta de la clase de cognición orientada a monitorear y a regular la propia cognición (Schraw y Gutiérrez, 2015). Así, desde otras posturas se asume que la metacognición hace referencia al conocimiento, la conciencia y la regulación del pensamiento (Zimmerman y Moylan, 2009). Históricamente se ha considerado como una categoría de estudio, algo difusa o borrosa, por la aparente dificultad que representa para los investigadores, en la práctica, diferenciar el grado de alcance cognitivo, del metacognitivo, que implica por sí mismo el desarrollo de la conciencia metacognitiva de un sujeto, que pueda ser consciente del estado de sus propios procesos cognitivos y de su aprendizaje. Esa es probablemente una de las principales razones por las cuales se consideran tan importantes los estudios en metacognición, porque se asumen como una puerta de entrada para la toma de conciencia y para impactar la propia capacidad de agencia de las personas (Balconi, 2010).

El constructo de conciencia metacognitiva ha sido desarrollado en el campo de la metacognición por Schraw y Dennison (1994), como una categoría de estudio integrada por al menos dos componentes esenciales: el conocimiento y la regulación. En la perspectiva de Schraw (2001), el conocimiento de la cognición se refiere a lo que las personas saben acerca de su propia cognición o acerca de la cognición en general. Incluye al menos tres tipos diferentes de conciencia metacognitiva: declarativa, procedimental y condicional (Brown, 1987, Jacobs y Paris, 1987; Schraw y Moshman, 1995). Mientras que la regulación metacognitiva se refiere a un conjunto de actividades que ayudan a los estudiantes a controlar su aprendizaje. La investigación apoya la suposición de que la regulación metacognitiva mejora el rendimiento de varias maneras, incluyendo un mejor uso de los recursos atencionales, y de las estrategias de aprendizaje existentes, así como una mayor conciencia de las dificultades en la comprensión (Brown y Palincsar, 1989; Cross y Paris, 1988). Aunque se han descrito varias habilidades regulatorias en la literatura (Schraw y Dennison, 1994), se reconocen al menos tres habilidades esenciales en todos los modelos de regulación: planificación, monitoreo y evaluación (Jacobs y Paris, 1987).

Diferentes investigadores han señalado altas correlaciones entre conocimiento y regulación metacognitiva (Balcikanli, 2011; Schraw y Dennison, 1994); así mismo, se ha asumido que el conocimiento metacognitivo, en general, es condición para las habilidades de regulación de la persona (Peña-Ayala y Cárdenas, 2015). Aunque la investigación sobre metacognición tiene una larga historia, como se mostrará en las siguientes secciones, la mayoría de los estudios se centran en los estudiantes de educación primaria y secundaria, así como en el abordaje de sus habilidades metacognitivas en campos de dominios académicos, no en la metacognición de los docentes. Por tanto, uno de los propósitos de la presente investigación fue examinar las diferencias en las habilidades metacognitivas del conocimiento (declarativo, procesal y condicional) y la regulación (planificación, monitoreo y evaluación), en función del tipo de desempeño profesional (estudiante universitario en práctica en los ciclos de básica y media académica; docente titular en los niveles de básica y media académica; docente universitario). 


\section{Marco teórico}

Varias teorías abordan el papel del autocontrol en el aprendizaje (Boekaerts y Rozendaal, 2010; De Bruin y Van Gog, 2012; Efklides, 2008; Winne y Nesbit, 2009). Sin embargo, Nelson y Narens (1990) propusieron un modelo de metacognición de dos procesos históricamente importantes que distingue entre procesos cognitivos, a nivel de objeto, y actividades metacognitivas, a nivel de meta. Este modelo postula un proceso de monitoreo que vincula la información en el nivel del objeto con los procesos de control autorreguladores en un nivel superior meta. La información obtenida mediante un monitoreo preciso se puede utilizar en el nivel meta para regular el aprendizaje posterior a nivel del objeto a través de procesos de control ejecutivo.

\section{Metacognición en docentes}

La categoría conciencia metacognitiva, generalmente, se ha empleado para hacer referencia al grado en el que una persona puede estar consciente en un momento dado, acerca de sus propios recursos cognitivos y metacognitivos disponibles, en función de agenciar su propio proceso de aprendizaje; así, algunos autores han empleado dicho constructo para nombrar el grado de conocimiento y regulación metacognitiva que la persona evidencia en un momento determinado, frente a una tarea específica de aprendizaje o de evaluación, así como también para referirse al proceso reflexivo derivado de su propio monitoreo metacognitivo en el estudio (Koriat, 2007; Schraw, 2001).

Específicamente, en relación con la importancia que tiene el hecho de que el docente sea un pensador metacognitivo, algunos autores reconocen que los docentes modelan su propia cognición, sin pensar, y sin impactar necesariamente la propia metacognición de sus estudiantes (Schraw, 2001), o sin emplear este monitoreo metacognitivo para mejorar su enseñanza, aun cuando se ha reconocido que tener un alto grado de conciencia metacognitiva es un aspecto importante para ser un docente altamente efectivo en la educación (Hart y Memnun, 2015; Kallio et al., 2017).

De este modo, a pesar de estar suficientemente documentada la importancia de la metacognición en el proceso de autorreflexión del docente frente a su enseñanza, se han realizado pocos estudios que aborden el desarrollo de una conciencia explícita de los docentes sobre su metacognición y sobre su propia capacidad para pensar, hablar y escribir en relación con su pensamiento como docente (Wilson y Bai, 2010; Zohar, 1999; 2006).

Dentro de los estudios previos en los que los investigadores han abordado el problema del conocimiento y la regulación de la metacognición del docente, en los que se han utilizado instrumentos de autorreporte, como el inventario de conciencia metacognitiva general (MAI, por su sigla en inglés) (Schraw y Dennison, 1994), o el inventario de conciencia metacognitiva para docentes (MAIT, por su sigla en inglés) (Balcikanli, 2011), se encuentran diferentes investigaciones en las que se ha indicado que los docentes reconocen que enseñar a los estudiantes a ser metacognitivos requiere una comprensión avanzada tanto del concepto de metacognición, como de las diferentes estrategias de pensamiento metacognitivo (Wilson y Bai, 2010). 
Desde esta perspectiva se encuentran estudios previos que resultan de interés en los que se ha evaluado el conocimiento y la regulación metacognitiva, y su correlación con otras variables implicadas en la relación pedagógica. Entre ellos se encuentra una tradición investigativa en la que se abordan:

Algunos estudios descriptivos en los que se han caracterizado las creencias sobre la enseñanza y el aprendizaje de docentes en áreas de dominio como la matemática en el nivel de primaria (Hart y Memnun, 2015), las actividades comunicativas en el aula de matemáticas en el nivel de secundaria (Raymond, 2019) y las pruebas de competencia docente propias de áreas de dominio como la biología (Ardi et al., 2018). Algunos estudios de correlaciones, en los que se ha establecido algunas asociaciones entre la metacognición del docente, con otras variables más psicológicas y de rendimiento asociadas al desempeño del docente, entre las que se encuentran la autoeficacia y el rendimiento académico, así como la edad y los años de experiencia profesional (Ghonsoolya et al., 2014; Stewart et al., 2007).

Entre algunos de los resultados encontrados en ambos tipos de estudios, descriptivos y correlacionales, se ha señalado lo siguiente. Primero, que el conocimiento de los profesores sobre la cognición y la regulación de los dominios específicos de la metacognición tienen un efecto predictor sobre sus creencias en torno a la enseñanza y el aprendizaje, en áreas de dominio como las matemáticas (Hart y Memnun, 2015). Segundo, se ha reconocido la importancia del conocimiento metacognitivo condicional de los docentes, para implementar actividades de comunicación en el aula, a fin de establecer cómo y cuándo usar la discusión y el trabajo escrito para involucrar a los estudiantes en preguntas, crear ideas y explicar su pensamiento (Raymond, 2019). Tercero, se ha señalado baja relación entre la capacidad metacognitiva y los resultados de las pruebas de competencia de los docentes en campos disciplinares como la biología (Ardi et al., 2018). Finalmente, sobre la correlación entre la conciencia metacognitiva del docente y otras variables, se ha descrito una alta asociación entre autoeficacia y metacognición, en la medida en que se considera que cuanto mayor es la autoeficacia del profesor, mayor es el uso que este puede hacer de las estrategias metacognitivas (Ghonsoolya et al., 2014), de igual forma, se ha encontrado asociación entre la mejora significativa del desempeño metacognitivo del docente, en relación con la edad y con los años de experiencia docente (Mai, 2015; Stewart et al., 2007).

En la revisión de antecedentes se evidencia que, a pesar de que algunos investigadores han señalado que la propia metacognición del docente afecta significativamente su proceso de enseñanza y el mismo aprendizaje del estudiante (Jiang et al., 2016; Prytula, 2008; 2012) $y$, aun cuando en diferentes estudios se reconoce la importancia del conocimiento y la regulación metacognitiva para la formación de docentes que reflexionen frente a su enseñanza y que puedan aportar en la formación de estudiantes que puedan autorregular su propio aprendizaje, son aún escasos los estudios en los que se establece el nivel de conciencia metacognitiva del docente y existen muchos menos reportes de dichos estudios en lengua hispana (Kallio et al., 2017).

\section{Metacognición y género}

Se constituyen en antecedentes específicos para la presente investigación algunos estudios en los que se han realizado descripciones del estado del conocimiento y de la regulación metacognitiva del docente, y su asociación con variables sociodemográficas como el género y 
Antonio Gutiérrez de Blume

Diana Marcela Montoya-Londoño

tipo de desempeño profesional (Sezgín y Akkaya, 2009), en los que se han encontrado resultados contradictorios que describen los grados de conocimiento y de regulación metacognitiva del docente, con hallazgos que reportan tanto altos como bajos niveles de desempeño en cada una de las subhabilidades metacognitivas evaluadadas (Mai, 2015; Palantis et al., 2017; Sezgín y Akkaya, 2009).

Así mismo, se reportan algunas investigaciones previas en las que no se encontraron asociaciones entre las puntuaciones del conocimiento y la regulación metacognitiva del docente, y el género, pero sí se lograron establecer diferencias con el nivel educativo del docente; este hallazgo permitió confirmar que los mejores desempeños en las diferentes variables metacognitivas evaluadas los presentaron los docentes que tenían el nivel de formación más avanzado (Cihanoglu, 2013; Ghonsoolya et al., 2014; Sezgín y Akkaya, 2009; Stewart et al., 2007). Finalmente, se registran mejores niveles de desempeño metacognitivo para docentes que han participado de procesos de formación metacognitiva en su preparación pedagógica durante largos periodos, y no solo como parte de un seminario de actualización docente de manera aislada (Ozturk, 2018).

La relación específica entre género y metacognición no se entiende bien, ya que la investigación sobre estos temas arroja resultados inconsistentes.

Ackerman et al. (2010), por ejemplo, descubrieron que el género era predictivo de una capacidad de conducir autoinformada más baja. Más específicamente, descubrieron que las mujeres subestimaban su capacidad de conducir autoevaluada, y que este efecto se mantuvo incluso después de controlar la capacidad de conducir previa. La investigación sobre el rendimiento en matemáticas reveló que los hombres no solo superaron a las mujeres en el rendimiento en matemáticas, sino que las mujeres se clasificaron a sí mismas con un rendimiento más bajo, y que este monitoreo metacognitivo del rendimiento mal calibrado en matemáticas,fue más pronunciado entre las mujeres (Chiu y Klassen, 2010; Özsoy, 2012; Sheldrake et al., 2014). Dentro de un contexto de física, Sharma y Bewes (2011) descubrieron que, aunque los hombres y las mujeres no diferían en sus logros en física, los hombres tendían a ser más precisos en sus índices de confianza de la mecánica, a pesar de que los puntajes de sesgo de las mujeres eran cercanos a los de los hombres, sugiriendo que generalmente entendieron su falta de comprensión de la mecánica. La amenaza de estereotipo fue una de las explicaciones plausibles que ofrecieron para el efecto de género.

Volz-Sidiropoulou y Gauggel (2012), en un estudio realizado con una muestra de adultos mayores (edad $\geq 65$ ), señalaron que las mujeres demostraron un mejor monitoreo metacognitivo que los hombres, ya que ellos presentaron un mayor sesgo y una mayor inexactitud en las medidas subjetivas, el retraso en el recuerdo y la tasa de olvido. Por el contrario, Nietfeld et al. (2014) no descubrieron un efecto de género significativo sobre el sesgo o la precisión del monitoreo, ya que los hombres y las mujeres calificaron la confianza de manera similar y exhibieron una precisión casi similar. Sin embargo, descubrieron que, dentro del análisis realizado en el caso de los hombres, los que tenían menos confianza superaban a sus contrapartes demasiado confiados.

Bussey y Bandura (1999) argumentaron que las diferencias de género observadas podrían deberse a la variación en la motivación y la autorregulación del aprendizaje, como resultado de las expectativas de género socialmente construidas. Por ejemplo, si se espera que los hombres superen a las mujeres en matemáticas y ciencias, el logro de los hombres en esas 
materias resulta de la presencia de factores motivacionales, como la autoeficacia percibida, que trabajan para involucrar habilidades de aprendizaje autorreguladas, como habilidades metacognitivas. Bembenutty (2007) también encontró evidencia de que la socialización de género cumple un papel en la autorregulación del aprendizaje. Es decir, fuera de las expectativas de logros específicos, se espera que las mujeres sean más organizadas y participen en una mayor regulación del esfuerzo que los hombres.

\section{El presente estudio}

De acuerdo con lo anterior, la presente investigación tuvo como objetivos establecer las diferencias en el conocimiento metacognitivo (declarativo, de procedimiento y condicional) y la regulación (planificación, monitoreo y evaluación) en una muestra de docentes colombianos, en función de las variables de género y tipo de desempeño profesional. De igual forma, el presente estudio buscó establecer correlaciones entre los componentes de la metacognición y las diferentes variables evaluadas.

\section{Método}

\section{Participantes, muestreo y diseño de investigación}

El estudio empleó un diseño de investigación descriptivo-correlacional, de carácter transversal (Hernández et al., 2010). La selección de la muestra se realizó de manera intencional, de acuerdo con los lineamientos metodológicos de un muestreo por conveniencia y según contactos establecidos con la unidad de calidad de la Alcaldía de Manizales y con una de las universidades públicas de la ciudad. Así, la muestra inicialmente estuvo conformada por 816 individuos; una vez fue depurada la base de datos para garantizar que los instrumentos hubieran sido por completo diligenciados, esta quedo conformada por 771 casos que fueron incluidos en el análisis final.

De estos 771 participantes, en relación con la distribución por género, se encontró que 388 eran mujeres, 382 hombres y 1 no indicado, y en relación con el tipo de desempeño profesional, 142 realizaban su labor profesional como docentes practicantes (último semestre de formación) en instituciones de educación básica y media académica, 326 eran docentes titulares en diferentes áreas de dominio en instituciones de educación básica y media académica, y 303 se desempeñaban en el contexto de la educación universitaria.

Todas las personas participaron en el estudio de forma voluntaria y se contó con la firma del consentimiento informado para su vinculación en el proceso de investigación.

\section{Materiales e instrumentos}

\section{Inventario de conciencia metacognitiva para docentes (MAIT-24)}

El MAIT es una versión adaptada para el contexto pedagógico del inventario para la evaluación de la conciencia metacognitiva en adultos (MAI) (Schraw y Sperling-Dennison, 1994) por Balcikanli (2011). Posteriormente, la versión original del mait en inglés fue traducida al castellano, adaptada culturalmente y validada por Gutiérrez de Blume y Montoya Londoño 
(2020) como el MAIT-24. De este modo, el instrumento permite medir la conciencia metacognitiva de los docentes sobre la enseñanza teniendo en cuenta el conocimiento y la regulación metacognitiva. El inventario consta de 24 ítems que se dividen en seis subescalas: conocimiento declarativo, conocimiento procedimental, conocimiento condicional, y planificación, monitoreo y evaluación.

El cuestionario se estructura con un formato de respuesta tipo Likert de 5 puntos, en el que el grado de acuerdo varía desde totalmente en desacuerdo (1) hasta totalmente de acuerdo (5). La puntuación se proporciona de la siguiente manera: totalmente en desacuerdo (1), en desacuerdo (2), neutral (3), de acuerdo (4), totalmente de acuerdo (5). Los resultados del alfa de Cronbach en las investigaciones alrededor del instrumento han permitido reconocer una alta confiabilidad para su uso en ciencias de la educación. Así, el alfa de Cronbach reportado para la prueba varía desde 0,79 , para el caso de la habilidad de evaluación, y va hasta 0,85, para el caso del conocimiento declarativo (Balcikanli, 2011). Para la presente muestra, los coeficientes de confiabilidad de consistencia interna, los alfa de Cronbach variaron de 0,78 a 0,86 para las seis escalas del MAIT-24 (ver anexo, para obtener una copia del instrumento MAIT-24 empleado en el presente estudio).

\section{Procedimiento}

Se realizó la presentación de los objetivos del estudio por parte del equipo de investigadores con cada grupo de docentes evaluados en tres momentos diferentes de trabajo, según el tipo de desempeño profesional (estudiante universitario en práctica en los ciclos de básica y media académica; docente titular en los niveles de básica y media académica; docente universitario). Igualmente, se realizó la firma del consentimiento informado por parte de los docentes que aceptaron participar voluntariamente en la investigación. Luego, se procedió a la aplicación del instrumento inventario de conciencia metacognitiva para docentes (alfa de Cronbach) (Balcikanli, 2011) en cada uno de los tres grupos participantes por separado.

\section{Análisis de los datos}

Antes de realizar el análisis de los datos, estos fueron probados para los supuestos estadísticos necesarios y seleccionados para valores atípicos univariados y multivariados. Se revisaron los datos de valores atípicos univariados por cada grupo de los análisis pertinentes para el presente estudio, género (hombre, mujer) y tipo de desempeño profesional (estudiante universitario en práctica en los ciclos de básica y media académica; docente titular en los niveles de básica y media académica; docente universitario).

Según la inspección de las parcelas de cajas y bigotes, de los 816 casos completos originales, se detectaron 45 que se consideraron valores atípicos univariantes en los cinco grupos. Por tanto, para evitar sesgos debido a puntajes extremos que de otro modo minarían la confiabilidad de los datos, se procedió a eliminar estos 45 casos de cualquier análisis posterior. Luego, se empleó la distancia de Mahalanobis y Cook's D como métricas para evaluar valores atípicos multivariados. Ninguno de los 771 casos restantes en la muestra se consideraron valores atípicos multivariados. Por consiguiente, todos los análisis principales continuaron con estos 771 casos restantes con datos completos. Los datos cumplieron con el supuesto de normalidad univariante (todos los valores de asimetría y curtosis fueron menores que el valor absoluto de 2) y la normalidad multivariada. Los datos también cumplieron con 
Antonio Gutiérrez de Blume

Diana Marcela Montoya-Londoño

Andrea Milena Osorio-Cárdenas

el supuesto de homogeneidad de la varianza del error (los valores $p$ de la prueba de Levene fueron todos mayores que 0,12 ) en el nivel univariante y la homogeneidad de las matrices de varianza-covarianza (valor $p$ de Box's $M=0,13$ ) en el nivel multivariante.

Se realizó un análisis de varianza factorial multivariante (Manova) de 2 (género: hombre, mujer) $\times 3$ (tipo de desempeño profesional: estudiante universitario en práctica en los ciclos de básica y media académica; docente titular en los niveles de básica y media académica; docente universitario). Las diversas habilidades metacognitivas (conocimiento declarativo, conocimiento de procedimiento, conocimiento condicional, planificación, monitoreo y evaluación) sirvieron como variables dependientes. El ajuste de Bonferroni a la significación estadística se utilizó para controlar la inflación de la tasa de error tipo I familiar, para los resultados ómnibus univariados $(0,05 / 6=0,008)$ y todas las comparaciones post hoc. Todos los tamaños de efecto para los resultados factoriales de Manova se informaron como $\eta^{2}$ parcial $\left(\eta^{2} p\right)$. Cohen (1988) especificó las siguientes pautas interpretativas para $\eta^{2}{ }_{p}: 0,010-.059$ como pequeño; 0,060-.139 como medio; y $\geq 0,140$ como grande.

\section{Resultados}

Las estadísticas descriptivas de la muestra por variable, género y tipo de desempeño profesional se presentan en la tabla 1. La tabla 2 muestra correlaciones bivariadas de orden cero para las variables metacognitivas por género. Por su parte, la tabla 3 detalla las correlaciones bivariadas de orden cero para las variables metacognitivas para los docentes practicantes en niveles de básica y media académica (en su último semestre de formación), y docentes titulares en niveles de básica y media académica; mientras que la tabla 4 hace lo mismo para los docentes universitarios.

\section{Tabla 1}

Datos descriptivos para conocimiento metacognitivo y variables de regulación según tipo de desempeño profesional y género

\begin{tabular}{|c|c|c|c|c|c|c|c|c|c|c|}
\hline \multirow[t]{2}{*}{ Variable } & \multicolumn{2}{|c|}{$\begin{array}{l}\text { Hombres } \\
(n=382)\end{array}$} & \multicolumn{2}{|c|}{$\begin{array}{l}\text { Mujeres } \\
(n=388)\end{array}$} & \multicolumn{2}{|c|}{$\begin{array}{l}\text { Docentes } \\
\text { practicantes } \\
\text { en niveles de } \\
\text { básica y } \\
\text { media } \\
\text { académica } \\
\qquad(n=142)\end{array}$} & \multicolumn{2}{|c|}{$\begin{array}{l}\text { Docentes } \\
\text { universitarios } \\
(n=303)\end{array}$} & \multicolumn{2}{|c|}{$\begin{array}{l}\text { Docentes } \\
\text { titulares en } \\
\text { niveles de } \\
\text { básica y } \\
\text { media } \\
\text { académica } \\
(n=326)\end{array}$} \\
\hline & $M$ & $S D$ & $M$ & $S D$ & $M$ & $S D$ & $M$ & $S D$ & $M$ & $S D$ \\
\hline$C D$ & 4,25 & 0,47 & 4,22 & 0,51 & 4,17 & 0,54 & 4,27 & 0,46 & 4,23 & 0,49 \\
\hline $\mathrm{CP}$ & 4,01 & 0,53 & 4,00 & 0,51 & 4,08 & 0,53 & 4,00 & 0,50 & 3,98 & 0,52 \\
\hline CC & 4,19 & 0,50 & 4,17 & 0,55 & 4,15 & 0,51 & 4,27 & 0,44 & 4,11 & 0,60 \\
\hline Planeación & 4,11 & 0,57 & 4,04 & 0,63 & 4,16 & 0,56 & 4,27 & 0,52 & 3,86 & 0,62 \\
\hline Monitoreo & 4,26 & 0,48 & 4,35 & 0,49 & 4,20 & 0,50 & 4,33 & 0,52 & 4,33 & 0,45 \\
\hline Evaluación & 4,17 & 0,53 & 4,29 & 0,54 & 4,19 & 0,52 & 4,19 & 0,56 & 4,29 & 0,49 \\
\hline
\end{tabular}


Clave: $\mathrm{CD}=$ conocimiento declarativo; $\mathrm{CP}=$ conocimiento procedural $; \mathrm{CC}=$ conocimiento condicional.

$$
n=771 .
$$

Fuente: elaboración propia

Es interesante observar que los participantes tienden a calificarse altamente en las variables metacognitivas, lo que sugiere que se consideran altamente conscientes de sus habilidades metacognitivas (tabla 1 ).

\section{Tabla 2}

Matriz de correlación bivariada de orden cero de variables metacognitivas por género.

\begin{tabular}{lllllll}
\hline Variable & 1 & 2 & 3 & 4 & 5 & 6 \\
\hline 1. CD & - & $0,52 * *$ & $0,50 * *$ & $0,40 * *$ & $0,46 * *$ & $0,37 * *$ \\
2. $\mathrm{cP}$ & $0,55 * *$ & - & $0,53 * *$ & $0,38 * *$ & $0,51 * *$ & $0,43 * *$ \\
3. cc & $0,51 * *$ & $0,58 * *$ & - & $0,47 * *$ & $0,49 * *$ & $0,37 * *$ \\
4. Planeación & $0,40 * *$ & $0,45 * *$ & $0,53 * *$ & - & $0,44 * *$ & $0,34 * *$ \\
5. Monitoreo & $0,44 * *$ & $0,38 * *$ & $0,47 * *$ & $0,54 * *$ & - & $0,69 * *$ \\
6. Evaluación & $0,37 * *$ & $0,33 * *$ & $0,35 * *$ & $0,42 * *$ & $0,65 * *$ & -
\end{tabular}

Nota: las correlaciones sobre la diagonal son para los hombres $(n=388)$ y las que están debajo de la diagonal son para las mujeres $(n=382)$.

$* * p<0,01$

Fuente: elaboración propia.

Tabla 3

Matriz de correlación bivariada de orden cero de variables metacognitivas para docentes practicantes en niveles de básica y media académica, y docentes titulares en niveles de básica y media académica.

\begin{tabular}{lllllll}
\hline Variable & 1 & 2 & 3 & 4 & 5 & 6 \\
\hline 1. CD & - & $0,53 * *$ & $0,43 * *$ & $0,39 * *$ & $0,44 * *$ & $0,36 * *$ \\
2. cp & $0,63 * *$ & - & $0,47 * *$ & $0,37 * *$ & $0,44 * *$ & $0,41 * *$ \\
3. cc & $0,63 * *$ & $0,67 * *$ & - & $0,46 * *$ & $0,47 * *$ & $0,36 * *$ \\
4. Planeación & $0,40 * *$ & $0,51 * *$ & $0,51 * *$ & - & $0,42 * *$ & $0,39 * *$ \\
5. Monitoreo & $0,40 * *$ & $0,49 * *$ & $0,54 * *$ & $0,63 * *$ & - & $0,64 * *$ \\
6. Evaluación & $0,34 * *$ & $0,34 * *$ & $0,35 * *$ & $0,40 * *$ & $0,68 * *$ & - \\
\hline
\end{tabular}


Nota: las correlaciones por encima de la diagonal son para el grupo de docentes de básica y media académica $(n=326)$ y las que están debajo de la diagonal son para el grupo de docentes practicantes en niveles de básica y media académica [en su último semestre de formación] $(n=142)$.

$* * p<0,01$

Fuente: elaboración propia.

\section{Tabla 4}

Matriz de correlación bivariada de orden cero de variables metacognitivas para docentes universitarios

\begin{tabular}{|c|c|c|c|c|c|c|}
\hline Variable & 1 & 2 & 3 & 4 & 5 & 6 \\
\hline 1. $C D$ & - & $0,50 * *$ & $0,56 * *$ & $0,47 * *$ & $0,47 * *$ & $0,39 * *$ \\
\hline 2. $\mathrm{cp}$ & & - & $0,63 * *$ & $0,44 * *$ & $0,45 * *$ & $0,38 * *$ \\
\hline 3. $\mathrm{CC}$ & & & - & $0,51 * *$ & $0,49 * *$ & $0,43 * *$ \\
\hline 4. Planeación & & & & - & $0,58 * *$ & $0,48 * *$ \\
\hline 5. Monitoreo & & & & & - & $0,70 * *$ \\
\hline 6. Evaluación & & & & & & - \\
\hline
\end{tabular}

$* * p<0,01$

$n=303$

Fuente: elaboración propia.

Con respecto a los patrones de correlación que se muestran en las tablas 2 a la 4, puede indicarse que, a pesar de que todas las correlaciones estaban en la dirección teóricamente esperada (es decir, positiva), en general, las más altas, según el tipo de desempeño profesional, tendían a estar en el grupo de docentes practicantes en niveles de básica y media académica y docentes titulares en niveles de básica y media académica.

Los resultados del Manova factorial $2 \times 3$ revelaron que la interacción género por tipo de desempeño profesional no fue estadísticamente significativa, $p=0,36$. Sin embargo, el efecto principal de género, multivariante $F(6,763)=4,34, p<0,001, \eta^{2} p=0,044$, y el tipo de efecto principal de tipo de desempeño profesional, multivariante $F(12,1528)=14,38, p<0,001, \eta^{2} p$ $=0,101$, alcanzaron significación estadística para la combinación lineal de variables dependientes. La interpretación de los resultados ómnibus univariados se expone a continuación.

El efecto principal de género fue estadísticamente significativo solo para el monitoreo, $F(1,768)=7,24, p=0,007, \eta^{2} p=0,014$, y evaluación, $F(1,768)=9,88, p=0,002, \eta^{2} p=$ 0,020 , después del ajuste de Bonferroni al valor $p$. Los resultados para el conocimiento declarativo, conocimiento de procedimiento, el conocimiento condicional y la planificación no 
fueron significativos, todos los valores de $p \geq 0,11$. Tanto el efecto principal del tipo de desempeño profesional -después del ajuste de Bonferroni-, como los resultados ómnibus univariados demostraron significación estadística para el conocimiento condicional, $F(2,768)$ $=7,87, p<0,001, \eta^{2} p=0,034$; planificación, $F(2,768)=44,16, p<0,001, \eta^{2} p=0,103 ; y$ monitoreo, $F(2,768)=6,14, p=0,001, \eta^{2} p=0,030$. Los resultados para el conocimiento declarativo, el conocimiento del procedimiento y la evaluación no alcanzaron significación estadística, todos los valores de $p \geq 0,04$.

Para el efecto principal de género, las mujeres reportaron puntajes significativamente más altos en monitoreo y evaluación que los hombres. Los resultados post hoc con el ajuste de Bonferroni para comparaciones múltiples mostraron que, con respecto al efecto principal del tipo de desempeño profesional, los docentes titulares en niveles de básica y media académica informaron significativamente menos conocimiento condicional, que los docentes universitarios. Un patrón similar fue evidente para la planificación, en el que los resultados post hoc mostraron que los docentes titulares en niveles de básica y media académica informaron significativamente menos habilidades de planificación, en comparación con los docentes practicantes en niveles de básica y media académica en su último semestre de formación y los docentes universitarios. Curiosamente, el patrón de monitoreo fue diferente, ya que los resultados post hoc revelaron que los docentes titulares en niveles de básica y media académica informaron una habilidad de monitoreo significativamente más alta que los docentes practicantes en niveles de básica y media académica en su último semestre de formación. Así mismo, se evidenció que los docentes universitarios reportaron una habilidad de monitoreo significativamente más alta, en comparación los docentes practicantes en niveles de básica y media académica en su último semestre de formación.

\section{Discusión}

El propósito del presente estudio fue examinar las diferencias grupales en las habilidades metacognitivas autoinformadas, según diferencias en el género y el tipo de desempeño profesional. Con respecto a las primeras, los resultados encontrados en la presente investigación se distancian de los hallazgos reportados en diferentes estudios realizados con docentes en los que no se establecen diferencias para ninguna de las habilidades de conocimiento y regulación metacognitiva entre hombres y mujeres (Ghonsoolya et al., 2014; Sezgín y Akkaya, 2009). Así, en el presente estudio las mujeres reportaron puntajes significativamente más altos que los hombres para las habilidades de regulación metacognitiva de monitoreo y evaluación. Al respecto, puede considerarse la perspectiva sobre metacognición general de Schraw y Moshman (1995) que puede hacerse extensiva también al campo de la enseñanza, desde la cual se indica que el monitoreo hace referencia a la supervisión en línea del curso de acción sobre una tarea de aprendizaje o de enseñanza. De esta manera, desde el rol del docente se consideran acciones de monitoreo, por ejemplo, la capacidad de realizar pruebas de autoevaluación mientras se enseña, o de forma concurrente con la tarea; mientras que la evaluación se refiere a la valoración de los procesos y productos cognitivos asociados a la enseñanza. Ejemplos de esta habilidad pueden ser la reevaluación de los objetivos y las conclusiones de una clase o de un curso.

En relación con el tipo de desempeño profesional, los hallazgos de la presente investigación permiten señalar que los docentes titulares en niveles de básica y media académica informaron significativamente menos conocimiento condicional que los docentes 
universitarios, y menos habilidades de planificación en comparación con los docentes practicantes en niveles de básica y media académica en su último semestre de formación y que los docentes universitarios.

Este hallazgo es consistente con diferentes investigaciones realizadas con estudiantes en los que se han indicado dificultades a nivel del conocimiento condicional, es decir, dificultades con relación a saber cuándo, dónde y por qué aplicar ciertas estrategias de acuerdo con las demandas de la tarea (Gutiérrez, 2014; Schraw y Dennison, 1994;). Esto podría interpretarse también en el contexto de la enseñanza, ya que se considera que la habilidad metacognitiva que debe tener el docente no es solo el conocimiento declarativo que tiene acerca de qué es la metacognición, sino también implica su propia habilidad metacognitiva puesta en acto en el diseño de las experiencias de aprendizaje que le brinda al estudiante (Ardi et al., 2018). De esta manera, el conocimiento metacognitivo supone saber cuándo y por qué emplear ciertos conocimientos y estrategias en la toma de decisiones en el aula frente a la enseñanza (Adadan y Oner, 2018). Este resultado pone en evidencia la importancia de generar espacios de formación permanente para los docentes titulares en básica y media académica que orientan su práctica profesional en estos niveles, en el fortalecimiento de competencias relacionadas con el conocimiento condicional, esto es, saber por qué y cuándo tomar decisiones y liderar acciones en función de agenciar y reflexionar sobre el propio proceso de enseñanza. De esta manera, en los primeros niveles de formación básica o secundaria, los estudiantes pueden empezar a asumir la metacognición como un factor importante en el aprendizaje efectivo, ya que les permite a las personas hacer seguimiento y organización de sus propios desempeños cognitivos (Schraw y Graham, 1997). En este sentido, se reconoce que un adecuado desempeño metacognitivo del estudiante aumenta el grado de conciencia en el aprendizaje, lo que permite emplear las estrategias de aprendizaje existentes de manera efectiva (Schraw y Graham, 1997; Sezgín y Akkaya, 2009).

De igual forma, en cuanto a las habilidades de regulación, los docentes titulares en niveles de básica y media académica también informaron significativamente menos habilidades de planificación, en comparación con los docentes practicantes en niveles de básica y media académica en su último semestre de formación y que los docentes universitarios. Este resultado es consistente con algunos estudios que han señalado que las dificultades de planeación de los docentes, en general, pueden estar relacionadas con el hecho de que el plan lo hacen en poco tiempo y no siempre de manera óptima (Ardi et al., 2018). Desde esta perspectiva, es importante considerar que las dificultades en la planeación pueden verse reflejadas en el contexto de la enseñanza, en cierta dificultad general para la selección de estrategias apropiadas y para la asignación de recursos cognitivos o materiales de acuerdo con el objetivo establecido y el requerimiento de la tarea, lo que podría implicar cierto compromiso en acciones como secuenciar estrategias y asignar tiempo y atención selectivamente antes de comenzar una tarea de enseñanza (Schraw y Moshman, 1995).

Los docentes titulares en niveles de básica y media académica informaron unas habilidades de monitoreo significativamente más altas, que los docentes practicantes en niveles de básica y media académica en su último semestre de formación y que los docentes universitarios, que ejercen su labor en el contexto de la educación superior. Este hallazgo, está probablemente relacionado con el hecho de que los docentes titulares de estos ciclos de formación (básica y media académica), quienes seguramente tienen por su trayectoria, más años de servicio y experiencia profesional, al enfocar su labor docente en los estudiantes más jóvenes, requieren 
realizar un proceso de monitoreo metacognitivo con mayor nivel de detalle, que aquellos docentes más noveles que tienen menos experiencia profesional (estudiantes de último nivel de formación en programas de educación) o que aquellos docentes que trabajan con adultos jóvenes (como sería el caso de los docentes universitarios). En este sentido, es importante señalar que los procesos de monitoreo ayudan el control autorregulador, lo que a su vez ayuda al aprendizaje (Gutierrez y Schraw, 2015) y, de manera extensiva, podría contribuir a ayudar también la reflexión sobre la enseñanza.

Al respecto, sería importante considerar la necesidad de empezar a desarrollar la habilidad metacognitiva de monitoreo frente al proceso de enseñanza, para el caso de los docentes en formación más jóvenes (estudiantes de último semestre de programas de licenciatura en educación), por cuanto los hallazgos del presente estudio confirman lo indicado en investigaciones previas en las que se reconoce la importancia del desarrollo de habilidades como el monitoreo y la evaluación en la formación de docentes, pues considera que cuando los docentes en formación supervisan su rendimiento, planifican las próximas sesiones, controlan cómo piensan, se autoevalúan y se autorregulan, tienen una mayor autoeficacia en su enseñanza (Ghonsoolya et al., 2014).

Entre los resultados, parece evidenciarse mejor desempeño en los docentes universitarios y los docentes practicantes en niveles de básica y media académica en su último semestre de formación, en relación con el conocimiento condicional y con la habilidad de planificación, que en los docentes titulares en niveles de básica y media académica. Esto podría indicar cierta relación teórica implícita, que a futuro habría que probar en la práctica, entre el proceso de formación, actualización y reflexión permanente que se vive en el contexto de la universidad, por su propia naturaleza y razón de ser, con el desarrollo de dichas habilidades metacognitivas en los docentes, dado que la mayoría de docentes de universidad, por su tipo de trabajo, deben tener estudios de posgrado al nivel de maestría y doctorado, y los estudiantes de último semestre de formación en el contexto de sus seminarios de práctica profesional tienen la oportunidad de desarrollar un pensamiento reflexivo frente a lo que pasa en el aula como parte de su currículo y créditos obligatorios en su formación.

De acuerdo con lo anterior, algunos investigadores han descrito la reflexión sobre la práctica de enseñanza del docente, como una forma de procesamiento activo y consciente de las experiencias, lo que les ha permitido señalar que cuando los docentes desarrollan habilidades metacognitivas de regulación (planificación, monitoreo y evaluación) con mayor frecuencia evidencian indicadores de un pensamiento reflexivo de alto nivel frente a su práctica de enseñanza (Adadan y Oner, 2014; Schraw, 2001).

En general, resulta de interés que los docentes practicantes en niveles de básica y media académica en su último semestre de formación se consideren altamente conscientes de sus habilidades metacognitivas, puesto que algunos investigadores en metacognición señalan que los profesionales en educación con más años de experiencia docente parecen evidenciar un desempeño más metacognitivo que aquellos que tienen menos experiencia docente (Jiang et al., 2016; Stewart et al., 2007). Este hallazgo resulta alentador debido a que parece evidenciar el interés actual, desde los niveles más iniciales de formación de docentes, por mejorar las habilidades de enseñanza metacognitiva de los nuevos docentes en formación y de aportar en la preparación de docentes reflexivos frente a su práctica profesional, mediante la generación de una formación encaminada a que los docentes más jóvenes puedan darse cuenta de sus fortalezas y debilidades en sus actividades de enseñanza. Este interés probablemente esté 
fundamentado en el reconocimiento de que la conciencia metacognitiva de los docentes es un medio para ayudar a los estudiantes a convertirse en aprendices autorregulados (Adams y Mabusela, 2014; Lee et al., 2015; Jiang et al., 2016; Robinson et al., 2001).

Así, las altas puntuaciones de los docentes más jóvenes y con menos experiencia, mediante las cuales se consideran a sí mismos altamente conscientes de sus habilidades metacognitivas, parece ser un hallazgo consistente con los fines de la educación colombiana y con las nuevas políticas nacionales de formación de docentes encaminadas al desarrollo de la capacidad crítica, reflexiva y analítica, mediante la cual se busca formar a los nuevos licenciados en educación con competencia para comprender, reflexionar, hacer seguimiento y tomar decisiones sobre los procesos de formación y enseñanza para favorecer los aprendizajes, la autorregulación y plantear acciones de mejora en los procesos educativos (Ministerio de Educación Nacional, 1994, 2015, 2017).

Las correlaciones bivariadas de orden cero revelaron que todas las correlaciones entre las habilidades metacognitivas estaban en la dirección teóricamente esperada, aunque había algunos patrones correlacionales interesantes entre la muestra completa y los diversos grupos por separado. A primera vista, se evidencia que las correlaciones fueron más altas para los docentes practicantes en niveles de básica y media académica en su último semestre de formación, y del grupo de los docentes universitarios, en comparación con los otros grupos. También, vale la pena mencionar que todas las correlaciones fueron pequeñas a grandes y estadísticamente significativas. Con respecto a los objetivos de la investigación, a pesar de que no se encontró evidencia de una interacción significativa de género por tipo de desempeño profesional, ambos efectos principales fueron estadísticamente significativos. Con respecto al género, las mujeres informaron calificaciones significativamente más altas para el monitoreo y la evaluación, lo que sugiere que las diferencias de género en las habilidades metacognitivas autoinformadas persisten (p. ej., Chiu y Klassen, 2010; Sharma y Bewes, 2011), aunque algunos estudios comienzan a no encontrar diferencias entre los géneros en las habilidades metacognitivas (Nietfeld et al., 2014).

\section{Conclusiones}

\section{Implicaciones para la teoría, la investigación y la práctica}

Entre los resultados se establecieron diferencias en la conciencia metacognitiva del docente, de acuerdo con el tipo de desempeño profesional, específicamente en relación con el conocimiento condicional y la planificación. Este resultado parece evidenciar rutas posibles de ajuste en el proceso de formación y actualización permanente que se les brinda a los docentes de los diferentes niveles. Así, queda claro que se requiere que a los docentes próximos a graduarse y a iniciar su vida profesional, desde la apuesta curricular que se les oferta en la universidad, se les brinden mayores oportunidades de formación para el desarrollo del monitoreo metacognitivo que luego puedan implementar en su enseñanza desde una perspectiva reflexiva, de la cual los primeros beneficiados van a ser los estudiantes de básica y media. Lo que también surge como necesario en el contexto del ejercicio profesional del docente universitario, en el que parece requerirse un mayor desarrollo de habilidades de monitoreo en la enseñanza en el marco del trabajo docente con adultos jóvenes. 
De igual forma, en los docentes titulares en niveles de básica y media académica, se requiere fortalecer el conocimiento condicional y la planificación, en función de saber cuándo, por qué y cómo usar el conocimiento profesional y pedagógico para potenciar las mejores oportunidades de aprendizaje de los estudiantes a su cargo, así como también, realizar procesos de planificación que permitan superar la eventual improvisación del ejercicio docente en el aula, que puede presentarse en algunos casos, lo que implica para el docente en ejercicio el desarrollo de habilidades para planear y monitorear actividades cognitivas y evaluar los resultados de estas actividades en función de su enseñanza (Ardi et al., 2018).

Desde el punto de vista teórico, en el presente estudio se evidenció que en la evaluación de la conciencia metacognitiva del docente se presentan diferencias por género y tipo de desempeño profesional del docente, diferencias que tiene sentido seguir explorando, puesto que parecen dar peso a la idea de una conciencia metacognitiva del docente, que puede ser limitada o potenciada por las oportunidades de formación y por los espacios para el desarrollo del pensamiento reflexivo que se generen en el contexto de la preparación profesional, posgradual o actualización permanente del docente. En concordancia con los resultados de la presente investigación, en relación con las diferencias en la conciencia metacognitiva de los docentes, de acuerdo con el género, resultados tan poco reportados en la literatura, sería conveniente avanzar en esta exploración realizando un estudio con una muestra más grande, y con réplica de este hallazgo en otros contextos sociales y culturales en Colombia y en otros países de habla hispana. Esto, tomando en cuenta que todos los hallazgos en los que no se reconocen ni se reportan diferencias de género corresponden a estudios realizados en países muy diferentes a la cultura latina, como Finlandia y Turquía, entre otros. Es probable que teóricamente puedan existir muchas diferencias en la conciencia metacognitiva del docente asociadas a la cultura, que deben explorarse en futuros estudios.

Así mismo, sería de interés continuar investigando sobre la relación entre algunas habilidades metacognitivas como el conocimiento condicional, la planificación y el monitoreo respecto al nivel educativo del docente y su relación con otras variables, como los años de experiencia docente o la edad del docente, así como el establecimiento de diferencias entre las habilidades metacognitivas, según las áreas de dominio, o diferencias en relación con la formación en el campo de las didácticas específicas en diferentes campos de la ciencia.

\section{Limitaciones de la presente investigación}

La presente investigación que involucró participantes humanos no está exenta de limitaciones. Primero, este estudio representa un diseño de investigación no experimental con un muestreo por conveniencia. Por tanto, esto limita las inferencias y conclusiones que pueden extraerse de los datos y también limita la generalización de los presentes hallazgos a otras muestras de la misma población.

En segundo lugar, el presente estudio empleó una encuesta de autoinforme para recopilar datos. Además del potencial para el sesgo de deseabilidad social, los participantes se calificaron a sí mismos altamente entre las variables bajo estudio, lo cual puede crear efectos de techo (ver tabla 1). Esto también limita las inferencias y conclusiones que se pueden extraer de los presentes resultados. Finalmente, es importante señalar que los tamaños del efecto para los resultados de Manova encontrados fueron todos pequeños y, por tanto, el 
Antonio Gutiérrez de Blume

Diana Marcela Montoya-Londoño Andrea Milena Osorio-Cárdenas

lector debe interpretar la importancia práctica de los hallazgos que se presentan con esto en mente.

A pesar de las diversas limitaciones del presente estudio, se resaltan dos puntos fuertes. Primero, la investigación se realizó en un entorno ecológicamente válido (es decir, en los entornos en los que estos participantes trabajan y aprenden), en vez del entorno artificial de un laboratorio; en consecuencia, las conclusiones que se presentan son más válidas contextualmente. En segundo lugar, se examinó la influencia de variables como el género y el tipo de desempeño profesional en las habilidades metacognitivas autoinformadas. Existe poca investigación hasta la fecha sobre estas relaciones, especialmente entre poblaciones de habla hispana como la muestra con la que se trabajó en el presente estudio. En consecuencia, este estudio contribuye de manera sustancial a una mejor comprensión de la compleja construcción de la metacognición.

A manera de síntesis, puede indicarse que en el presente estudio se encontraron diferencias significativas entre hombres y mujeres en el monitoreo y la evaluación, ambas habilidades de regulación, pero no en las habilidades de conocimiento metacognitivo (declarativo, procedimental y condicional). Las mujeres informaron habilidades significativamente mayores de monitoreo y evaluación en comparación con los hombres; resultado que parece consistente con el hecho de que, en muchas culturas, las niñas todavía están socializadas y condicionadas para ejercer la profesión docente en una relación probablemente desproporcionada con respecto a los niños (Chiu y Klassen, 2010). Así mismo, las diferencias encontradas en relación con el género son consistentes con estudios previos que han postulado que las mujeres, en comparación con los hombres, hacen un uso mayor de estrategias de autorregulación, y tienen mejores habilidades de automonitoreo, autocontrol, establecimieto de metas, planificación, organización de materiales de estudio y mejores habilidades para la autorreflexión en torno al aprendizaje (Parra et al., 2014; Tang y Neber, 2008). Sería interesante investigar si esta tendencia se mantendría cuando más hombres se conviertan en docentes. Sin embargo, es importante tener en cuenta que los efectos de estas diferencias de género fueron bastante pequeños $y$, por consiguiente, se necesitan estudios de replicación para evaluar la estabilidad de los presentes hallazgos.

Con respecto al tipo de desempeño profesional, los docentes titulares en niveles de básica y media académica informaron niveles significativamente más bajos de conocimiento condicional y planificación, aunque también informaron niveles significativamente más altos de monitoreo en comparación con los otros dos grupos evaluados. Estos resultados mixtos respaldan la necesidad de desarrollar programas de capacitación que modelen y fomenten explícitamente, el aprendizaje y la aplicación de habilidades metacognitivas, como las que se consideraron de interés para este estudio. Las sociedades no pueden esperar que los docentes enseñen, modelen e incentiven el aprendizaje y la aplicación de habilidades metacognitivas en su práctica profesional cotidiana, cuando ellos mismos carecen de dicha capacitación. En este sentido, algunas investigaciones han señalado el perfeccionamiento de las habilidades metacognitivas en el desarrollo profesional de los docentes, como una forma de mejorar la calidad de la educación y de lograr la formación de profesores exitosos (Sanabria et al., 2014). 


\section{Referencias}

Ackerman, M., Crowe, M., Vance, D., Wadley, V., Owsley, C. y Ball, K. (2010). The impact of feedback on self-rated driving ability and driving self-regulation among older adults. The Gerontologist, 51(3), 367-378. 10.1093/geront/gnq082

Adadan, E. y Oner, D. (2018). Examining preservice teachers' reflective thinking skills in the context of web-based portfolios: The role of metacognitive awareness. Australian Journal of Teacher Education, 43(11), 26-50. https://search.informit.com.au/documentSummary; dn=107182555493753;res=IELA PA

Adams, J. y Mabusela, M. (2014). A metacognitive approach to teacher development: Supporting national professional diploma in education (Npde) students. Mediterranean Journal of Social Sciences, 5(15), 289-296. 10.5901/mjss.2014.v5n15p289

Ardi, A., Fadilah, M. e Ichsani, B. (2018). Metacognitive ability relationship with test result of senior high school of biology teacher competence in Sijunjung District. IOP Conference Series: Materials Science and Engineering, 335, 1-7. 10.1088/1757899X/335/1/012086

Balcikanli, C (2011). Inventario de conciencia metacognitiva para docentes (MAIT). Electronic Journal of Research in Educational Pychology, 9(3), 1309-1332. 10.25115/ejrep.v9i25.1620

Balconi, M. (2010). Neuropsychology of the sense of agency. Springer.

Bembenutty, H. (2007). Self-regulation of learning and academic delay of gratification: Gender and ethnic differences among college students. Journal of Advanced Academics, 18(4), 586-616. https://doi.org/10.4219/jaa-2007-553

Boekaerts, M. y Rozendaal, J. S. (2010). Using multiple calibration measures in order to capture the complex picture of what affects students' accuracy of feeling of confidence. Learning and Instruction, 20(5), 372-382. 10.1016/j.learninstruc.2009.03.002

Brown, A. (1987). Metacognition, executive control, self-regulation, and other more mysterious mechanisms. En F. Weinert y R. Kluwe (eds.), Metacognition, motivation, and understanding (pp. 65-116). Erlbaum.

Brown, A. y Palincsar, A. (1989). Guided, cooperative learning and individual knowledge acquisition. En L. B. Resnick (ed.), Knowing and learning: Essays in honor o f Robert Glaser. (pp. 393-451). Erlbaum.

Bussey, K. y Bandura, A. (1999). Social cognitive theory of gender development and differentiation. Psychological Review, 106(4), 676-713. https://doi.org/10.1037/0033295X.106.4.676

Cihanoglu, M. (2013). Metacognitive awareness of teacher candidates. Procedia. Social and Behavioral Sciences, 46, 4529-4533. 10.1016/j.sbspro.2012.06.290

Cohen, J. (1988). Statistical power analysis for the behavioral sciences. (2a. ed.). Lawrence Earlbaum \& Associates. https://doi.org/10.4324/9780203771587 
Cross, D. y Paris, S. (1988). Developmental and instructional analyses of children's metacognition and reading comprehension. Journal of Educational Psychology, 80(2), 131-142. 10.1037/0022-0663.80.2.131

Chiu, M. y Klassen, R. (2010). Relations of mathematics self-concept and its calibration with mathematics achievement: Cultural differences among fifteen-year-olds in 34 countries. Learning and Instruction, 20(1), 2-17. 10.1016/j.learninstruc.2008.11.002

De Bruin, A. B. H. y Van Gog, T. (2012). Improving self-monitoring and self-regulation: From cognitive psychology to the classroom. Learning and Instruction, 22(4), 245-252. $10.1016 /$ j.learninstruc.2012.01.003

Efklides, A. (2008). Metacognition: Defining its facets and levels of functioning in relation to self-regulation and co-regulation. European Psychologist, 13(4), 277-287. 10.1027/1016-9040.13.4.277

Ghonsoolya, B., Hassan Khajavyb, G. y Mohaghegh Mahjoobi, F. (2014). Self-efficacy and metacognition as predictors of Iranian teacher trainees' academic performance: A path analysis approach. Procedia. Social and Behavioral Sciences, 98, 590-598. $10.1016 /$ j.sbspro.2014.03.455

Gutiérrez, A. P. (2014). Comprehension monitoring: The role of conditional knowledge. https://www.improvewithmetacognition.com/comprehension-monitoring-the-role-ofconditional-knowledge/

Gutiérrez, A. P. y Schraw, G. (2015). Effects of strategy training and incentives on students' performance, confidence, and calibration. The Journal of Experimental Education, 83(3), 386-404. 10.1080/00220973.2014.907230

Gutiérrez de Blume, A. P. y Montoya Londoño, D. M. (2020). El inventario de conciencia metacognitiva para docentes (MAIT): adaptación cultural y validación en una muestra de docentes colombianos. Psychologia, 14(1), 115-130. 10.21500/19002386.4584

Hart, L. y Memnun, D. (2015). The relationship between preservice elementary mathematics teachers' beliefs and metacognitive awareness. Journal of Education and Training Studies, 3(5). 70-77. 10.11114/jets.v3i5.840

Hernández, R., Fernández, C. y Baptista, P. (2014). Metodología de la investigación. McGrawHill.

Jacobs, J. y Paris, S. (1987). Children's metacognition about reading: Issues in definition, measurement, and instruction. Educational Psychologist, 22(3), 255-278. $10.1080 / 00461520.1987 .9653052$

Jiang, Y., Ma, L. y Gao, L. (2016). Assessing teachers' metacognition in teaching: The teacher metacognition inventory. Teaching and Teacher Education, 59, 403-413. $10.1016 / j$.tate. 2016.07 .014

Kallio, H., Virta, K., Kallio, M., Virta, A., Hjardemaal, F. y Sandven, J. (2017). The utility of the metacognitive awareness inventory for teachers among in-service teachers. Journal of Education and Learning, 6(4), 78-91. 10.5539/jel.v6n4p78

Koriat, A. (2007). Metacognition and consciousness. En P. Zelano, M. Moscovitch y E. Thompson (eds.), The Cambridge Handbook of Consciousness (pp. 289-25). Cambridge University Press. 
Lee, S., Irving, K., Pape, S. y Owens, D. (2015). Teachers' use of interactive technology to enhance students' metacognition: Awareness of student learning and feedback. Journal of Computers in Mathematics \& Science Teaching, 34(2), 175-198. https://www.learntechlib.org/primary/p/114753/

Mai, M (2015). Science teachers' self-perception about Metacognition. Journal of Educational and Social Research, 5(1), 77-86. 10.5901/jesr.2015.v5n1s1p77

Ministerio de Educación Nacional (MEN). (1994). Ley General de Educación. https://www.mineducacion.gov.co/1621/articles-85906_archivo_pdf.pdf

Ministerio de Educación Nacional (MEN). (2015). Decreto 2450. https://www.mineducacion.gov.co/1759/articles-357048_recurso_1.pdf

Ministerio de Educación Nacional (MEN). (2017). Resolución 18583. https://www.usbcali.edu.co/sites/default/files/resolucion_final_18583_de_2017derog a_2041.pdf

Nelson, T. O. y Narens, L. (1990). Metamemory: A theoretical framework and new findings. En G. Bower (ed.), The psychology of learning and motivation (vol. 26, pp. 125-173). Academic Press.

Nietfeld, J., Shores, L. y Hoffman, K. (2014). Self-regulation and gender within a game-based learning environment. Journal of Educational Psychology, 106(4), 961-973. $10.1037 / \mathrm{a} 0037116$

Özsoy, G. (2012). Investigation of fifth grade students' mathematical calibration skills. Educational Sciences: Theory and Practice, 12(2), 1190-1194.

Ozturk, N. (2018). The relation between teachers' self-reported metacognitive awareness and teaching with metacognition. International Journal of Research in Teacher Education, 9(2), 26-35.

Palantis, N., Mohamed, J., Ibrahim, A., Ismail, S., Anuar, N., Ma'rof, A. y Buang, N. (2017). Patterns of metacognitive awareness among primary school teachers. Varia Pendidikan, 29(2), 141-146. http://journals.ums.ac.id/index.php/varidika/article/view/5629

Parra, J., Cerda, C., López-Vargas, O. y Saiz, J. L. (2014). Género, autodirección del aprendizaje y desempeño académico en estudiantes de pedagogía. Revista Educación y Educadores, 17(1), 91-107. https://www.redalyc.org/pdf/834/83430693005.pdf

Peña-Ayala, A. y Cárdenas, L. (2015). A conceptual model of the metacognitive activity. En A. Peña-Ayala (ed.), Metacognition: Fundaments, applications and trends. A prolife of the current state -of-the-art (pp. 39-64). Springer.

Prytula, M. P. (2008). Scholarship epistemology: An exploratory study of teacher metacognition within the context of successful learning communities [Tesis doctoral]. https://pdfs.semanticscholar.org/1756/7a36be0bdb39cf1c02931be5bb6e3661e059.p $\mathrm{df}$

Prytula, M. P. (2012). Teacher metacognition within the professional learning community. International Education Studies, 5(4), 112-121. 10.5539/ies.v5n4p112 
Raymond, K. M. (2019). First-year secondary mathematics teachers' metacognitive knowledge of communication activities. Investigations in Mathematics Learning, 11(3), 167-179. 10.1080/19477503.2018.1425590

Robinson, E., Anderson-Harper, H. y Kochan, F. (2001). Strategies to improve reflective teaching. Journal of Pharmacy Teaching, 8(4), 49-58. 10.1300/J060v08n04_04

Sanabria, L., López, O. y Leal, S. (2014). Desarrollo de competencias metacognitivas e investigativas en docentes en formación mediante la incorporación de tecnologías digitales: aportes a la excelencia docente. Revista Colombiana de Educación, 67, 147170. https://revistas.pedagogica.edu.co/index.php/RCE/article/view/2875

Schraw, G. (2001). Promoting general metacognitive awareness. En H. Hartman (ed.), Metacognition in learning and instruction. Theory, research and practice (pp. 3-16). Springer Science.

Schraw, G. y Graham, T. (1997). Helping gifted students develop metacognitive awareness. Roeper Review, 20, 4-8. 10.1080/02783199709553842

Schraw, G. y Gutiérrez, A. (2015). Metacognitive strategy instruction that highlinghts the role of monitoring and control processes. En A. Peña (ed.), Metacognition: Fundaments, applications, and trends (pp. 3-15). Springer International.

Schraw, G. y Moshman, D. (1995). Metacognitive theories. Educational Psychology Review, 7(4), 351-371. 10.1007/BF02212307

Schraw, G. y Dennison, R (1994). Assessing metacognitive awareness. Contemporany Educational Psychology, 19, 460-475. 10.1006/ceps.1994.1033

Sezgín, D. y Akkaya, R. (2009). The levels of metacognitive awareness of primary teacher trainees. Procedia. Social and Behavioral Sciences, 1, 1919-1923. 10.1016/j.sbspro.2009.01.337

Sharma, M. D. y Bewes, J. (2011). Self-monitoring: Confidence, academic achievement and gender differences in physics. Journal of Learning Design, 4(3), 1-13. $10.5204 /$ jld.v4i3.76

Sheldrake, R., Mujtaba, T., y Reiss, M. (2014). Calibration of self-evaluations of mathematical ability for students in England aged 13 and 15, and their intentions to study noncompulsory mathematics after age 16. International Journal of Educational Research, 64, 49-61. 10.1016/j.ijer.2013.10.008

Stewart, P., Cooper. S. y Moulding, L. (2007). Metacognitive development in professional educators. The Researcher, 21(1), 32-40. http://www.nrmera.org/wpcontent/uploads/2016/02/Researcherv21n1Stewart.pdf

Tang, M. y Neber, H. (2008). Motivation and self-regulated science learning in high-achieving students: Differences related to nation, gender, and grade-level. High Ability Studies, 19(2), 103-116. 10.1080/13598130802503959

Volz-Sidiropoulou, E. y Gauggel, S. (2012). Do subjective measures of attention and memory predict actual performance? Metacognition in older couples. Psychology and Aging, 27(2), 440-450. https://doi.org/10.1037/a0025384 
Wilson, N. y Bai, H. (2010). The relationships and impact of teachers' metacognitive knowledge and pedagogical understandings of metacognition. Metacognition Learning, 5, 269-288. 10.1007/s11409-010-9062-4

Winne, P. H. y Nesbit, J. C. (2009). Supporting self-regulated learning with cognitive tools. En D. J. Hacker, J. Dunlosky y A. C. Graesser (eds.), Handbook of metacognition in education (pp. 259-277). Routledge/Taylor \& Francis Group.

Zimmerman, B. J. y Moylan, A. R. (2009). Self-regulation: Where metacognition and motivation intersect. En D. Hacker, J. Dunlosky y A. Graesser (eds.), Handbook of metacognition in education (pp. 299-315). Routledge.

Zohar, A. (1999). Teachers' metacognitive knowledge and the instruction of higher order thinking. Teaching and Teacher Education, 15, 413-429. 10.1016/S0742051X(98)00063-8

Zohar, A. (2006). The nature and development of teachers' metastrategic knowledge in the context of teaching higher order thinking. The Journal of Learning Sciences, 15(3), 331-377. 10.1207/s15327809jls1503_2 


\section{Anexo}

Versión original del MAIT para docentes de habla hispana (MAIT-24) de Gutiérrez de Blume y Montoya Londoño (2020), publicado por primera vez en la revista Psychologia.

El instrumento MAIT es una lista de 24 declaraciones. No hay respuestas correctas o incorrectas en esta lista de afirmaciones acerca de usted mismo como docente. Sus respuestas expresan simplemente lo que es verdad para usted, o lo que usted considera importante en su ejercicio como docente. Lea cuidadosamente cada declaración y elija la opción que mejor lo describe.

Muchas gracias por su participación.

Opciones de respuesta:

1 = totalmente en desacuerdo, 2 = en desacuerdo, $3=$ neutral, $4=$ de acuerdo, $5=$ muy de acuerdo

\begin{tabular}{|c|c|c|c|c|c|}
\hline $\begin{array}{l}\text { 1. Soy consciente de las fortalezas y debilidades a la hora } \\
\text { de enseñar. }\end{array}$ & 1 & 2 & 3 & 4 & 5 \\
\hline $\begin{array}{l}\text { 2. Trato de utilizar técnicas de enseñanza que me } \\
\text { funcionaron en el pasado. }\end{array}$ & 1 & 2 & 3 & 4 & 5 \\
\hline $\begin{array}{l}\text { 3. Utilizo mis fortalezas para compensar mis debilidades en } \\
\text { el proceso de enseñanza. }\end{array}$ & 1 & 2 & 3 & 4 & 5 \\
\hline $\begin{array}{l}\text { 4. Regulo mi propio ritmo de trabajo para tener el tiempo } \\
\text { suficiente. }\end{array}$ & 1 & 2 & 3 & 4 & 5 \\
\hline $\begin{array}{l}\text { 5. Periódicamente me preguntó, si estoy cumpliendo mis } \\
\text { objetivos mientras estoy enseñando. }\end{array}$ & 1 & 2 & 3 & 4 & 5 \\
\hline $\begin{array}{l}\text { 6. Me pregunto qué tan bien he logrado mis objetivos de } \\
\text { enseñanza, una vez que he terminado. }\end{array}$ & 1 & 2 & 3 & 4 & 5 \\
\hline $\begin{array}{l}\text { 7. Sé qué habilidades son las más importantes para poder } \\
\text { ser un buen docente. }\end{array}$ & 1 & 2 & 3 & 4 & 5 \\
\hline $\begin{array}{l}\text { 8. Tengo una razón específica para elegir cada una de las } \\
\text { técnicas de enseñanza que utilizo en clase. }\end{array}$ & 1 & 2 & 3 & 4 & 5 \\
\hline $\begin{array}{l}\text { 9. Puedo motivarme a mí mismo cuando debo orientar una } \\
\text { clase. }\end{array}$ & 1 & 2 & 3 & 4 & 5 \\
\hline $\begin{array}{l}\text { 10. Establezco mis propias metas de enseñanza antes de } \\
\text { comenzar una clase. }\end{array}$ & 1 & 2 & 3 & 4 & 5 \\
\hline $\begin{array}{l}\text { 11. Generalmente evalúo qué tan útiles son mis técnicas de } \\
\text { enseñanza mientras oriento una clase }\end{array}$ & 1 & 2 & 3 & 4 & 5 \\
\hline $\begin{array}{l}\text { 12. Generalmente me pregunto si pude haber utilizado } \\
\text { otras técnicas de enseñanza, después de cada clase. }\end{array}$ & 1 & 2 & 3 & 4 & 5 \\
\hline
\end{tabular}


Antonio Gutiérrez de Blume

Diana Marcela Montoya-Londoño

\begin{tabular}{|c|c|c|c|c|c|}
\hline 13. Tengo control sobre qué tan bien enseño. & 1 & 2 & 3 & 4 & 5 \\
\hline $\begin{array}{l}\text { 14. Soy consciente de qué técnicas de enseñanza utilizo } \\
\text { mientras estoy enseñando. }\end{array}$ & 1 & 2 & 3 & 4 & 5 \\
\hline $\begin{array}{l}\text { 15. Dependiendo de la situación, utilizo diferentes técnicas } \\
\text { de enseñanza. }\end{array}$ & 1 & 2 & 3 & 4 & 5 \\
\hline $\begin{array}{l}\text { 16. Me cuestiono acerca del material de enseñanza que voy } \\
\text { a utilizar en clase. }\end{array}$ & 1 & 2 & 3 & 4 & 5 \\
\hline $\begin{array}{l}\text { 17. Generalmente reviso qué tanto han comprendido mis } \\
\text { estudiantes el tema, mientras estoy enseñando. }\end{array}$ & 1 & 2 & 3 & 4 & 5 \\
\hline $\begin{array}{l}\text { 18. Después de orientar una clase, me pregunto si podría } \\
\text { hacerlo mejor en una próxima ocasión }\end{array}$ & 1 & 2 & 3 & 4 & 5 \\
\hline 19. Sé qué debo enseñar. & 1 & 2 & 3 & 4 & 5 \\
\hline 20. Empleo técnicas de enseñanza útiles automáticamente. & 1 & 2 & 3 & 4 & 5 \\
\hline $\begin{array}{l}\text { 21. Sé cuándo cada técnica de enseñanza que utilizo será } \\
\text { más eficaz. }\end{array}$ & 1 & 2 & 3 & 4 & 5 \\
\hline $\begin{array}{l}\text { 22. Organizo mi tiempo para lograr mis metas de } \\
\text { enseñanza. }\end{array}$ & 1 & 2 & 3 & 4 & 5 \\
\hline $\begin{array}{l}\text { 23. Me hago preguntas acerca de qué tan bien lo estoy } \\
\text { haciendo, mientras estoy enseñando. }\end{array}$ & 1 & 2 & 3 & 4 & 5 \\
\hline $\begin{array}{l}\text { 24. Me hago preguntas acerca de si he considerado todas } \\
\text { las técnicas posibles después de dar una clase }\end{array}$ & 1 & 2 & 3 & 4 & 5 \\
\hline
\end{tabular}

